\title{
Strategic Management of Other-Provided Information Online: Personality and Network Variables
}

\author{
Jian Rui, M.A. \\ University at Buffalo \\ jianrui@buffalo.edu
}

\author{
Michael A. Stefanone, Ph.D. \\ University at Buffalo \\ ms297@buffalo.edu
}

\begin{abstract}
Effective self-presentation is challenging in the contemporary computer-mediated communication environment due to the abundance of other-provided information. This study compared two approaches, one that focuses on internal factors (the personality approach) and the other environmental factors (the network approach), explaining why individuals engage in strategic self-presentation to manage other-provided information on social network sites. Using an online survey $(N=322)$ of participants from the United States and Singapore, results suggest that the two approaches work differently in different contexts, depending on the communication mode. Specifically, individuals who stake self-esteem on physical appearance and have diverse online networks are more strategic in their maintenance of other-provided information. Practical and theoretical implications about online selfpresentation are provided.
\end{abstract}

\section{Introduction}

Self-presentation is a process in which individuals control how they are perceived by others [18]. In order to construct positive public images, individuals tend to selectively present themselves by displaying their most positive individual characteristics. However, this selective self-presentation is increasingly difficult in contemporary computer-mediated communication (CMC) environments because Internet-based communication tools enable users to interact with each other. These interactions reduce users' control over the information about themselves online [22] because information provided by others (referred to as otherprovided information) can be inconsistent with the idealized image users construct. As users have limited control over other-provided information, it tends to be more credible and thus may have a greater impact than information provided by users themselves on how they are perceived [35].
Although recent research examined factors that influence strategic self-presentation in response to unwanted other-provided information [25, 27], two theoretical gaps are identified. First, the literature provides two different theoretical frameworks about self-presentation. One framework emphasizes the impact of internal factors on self-presentation $[17,18]$. Researchers argue that internal factors such as personality are the cause of selective self-presentation behavior. This explanation is in contrast to the traditional theoretical framework that defines selfpresentation as a function of self-audience interaction in which individuals adjust their behaviors based on the expectations of specific audiences [11]. Although researchers still regard internal factors, they argue that the external environment drives strategic selfpresentation behavior [17]. Both frameworks were empirically tested, which provides better explanations for strategic self-presentation behaviors may depend on the context in which they occur. Therefore, better understanding this issue contributes to understanding strategic impression management behavior on SNS.

In addition, researchers adopting the approach emphasizing internal factors governing selfpresentation argue this behavior can be explained from the perspective of self-esteem [17]. Humans tend to seek approval because recognition from others can boost their self-esteem. Thus, they are motivated to present themselves in a positive light to ensure they are publicly recognized [17]. While self-esteem was traditionally defined as a global concept, recent research shows that individuals look to different domains of their life for specific appraisals of selfesteem [8]. Research found that individuals staking self-esteem on external recognition are increasingly motivated to engage in strategic management of otherprovided information [25]. However, it is unclear which domain of public sphere contingencies of selfworth (CSW) (for example, appearance) has the greatest impact on strategic self-presentation behavior.

Therefore, the current research proposes to compare the factors that influence strategic management of other-provided information online. 
Drawing upon the two-component model of impression management [18], the dramaturgical approach to selfpresentation [11], and recent research on CSW [5, 8], we investigate whether internal factors- herein referred to as the personality approach, or external environmental characteristics- referred to as the network approach, have a stronger impact on strategic self-presentation behaviors online. It remains unclear which perspective better explains self-presentation. Thus, both frameworks were tested empirically.

\section{The personality approach}

\subsection{The two-component model of impression management}

Leary and Kowalski argue that two main subprocesses underlie self-presentation: impression motivation and impression construction. The former refers to how individuals are motivated to manage their impressions by others, whereas the latter refers to what factors influence their construction of specific images [18]. The motivation for self-presentation depends on three factors: whether individual goals are related to public impressions, what value desired outcomes have, and whether current images are discrepant from desired images.

Online, individuals have different objectives when using Internet-based communication tools like social network sites (SNS). Most users seek to be socially accepted [32], heavy users compete for attention [30], and newcomers are motivated to integrate into new surroundings and cultivate resources like social capital [9, 19]. All these goals are contingent upon constructing and maintaining positive self-images because negative impressions are generally associated with social rejection [3, 32]. Importantly, individuals differ on the dimensions of self they value when constructing and maintaining their self-image. In other words, individuals employ differing contingencies in the assessment of their own self-worth.

\subsection{Contingencies of self-worth}

Although self-esteem is a commonly used measure in social psychology, it has a range of different conceptualizations in the literature. One approach defines self-esteem as a global judgment of the value of self [24]. Alternatively, esteem can also be treated as a domain-specific evaluation of one's self [36]. For example, some people base their self-worth on contingencies like their appearance or academic competence. There is a growing body of research that shows different individuals look to different domains of their life for self-esteem appraisals. Therefore, valid measurement of self-esteem requires a multidimensional approach. As individuals enact behaviors that enhance their self-worth within particular contingent domains [8], contingencies of self-worth (CSW) was proposed and conceptualized as domains or categories of "outcomes on which a person has staked his or her self-esteem, so that person's view of his or her value or worth depends on perceived successes or failures or adherence to self-standards in that domain" (p. 594).

Seven contingencies have been identified, including competencies, competition, approval from generalized others, family support, appearance, God's love, and virtue [5]. Research shows that CSW explains behaviors in a variety of contexts. For example, appearance CSW predicted the extent to which college students socialized, shopped, joined social groups such as fraternities/sororities, and engaged in grooming behaviors [5]. However, appearance CSW had a negative relationship with spiritual activities and time spent with family [4]. Further, academic competency demonstrated a positive relationship with time studying and a negative relationship with socializing [7]. Regardless of high or low overall self-esteem, people seek positive feelings associated with success in their CSW and avoid negative feelings associated with failure in these domains.

Researchers categorized CSW into externally- and internally-oriented domains [5, 8]. Individuals with externally-oriented CSW stake their self-esteem on outside evaluations such as physical appearance, competence, and others' approval, whereas individuals with internally-oriented CSW stake their self-esteem on more private dimensions including religion, family, and virtue. This two-factor structure was confirmed in a study about CSW and online behavior [31]. Once again, the first factor was comprised of elements related to more traditional, personal domains like family, virtue, and God's love, and was labeled private sphere contingencies. The second factor focused on approval, appearance and competition, labeled as public sphere contingencies. These public-sphere contingencies relate more to public interaction and evaluation, and their results show that public sphere CSW was associated with increased photo sharing online and a generally higher level of competition for attention on social network sites [31].

In the context of the current research, most goals motivating SNS uses described above, including social acceptance, identity confirmation, and attention seeking, are based on gaining recognition from personal/online network members. Because individuals exhibiting greater stakes in public sphere CSW base their self-esteem on recognition from others, these 
impression-based goals should function as primary motivators.

Although idealized self-images are valuable and facilitate the attainment of impression-based goals for SNS users with public sphere CSW, it is becoming more difficult for users to maintain the idealized selfimages they create online today. As online networks become more expansive and inclusive, the abundance of other-provided information functionally reduces the level of control users have over information available about themselves. Thus, discrepancies between idealized and current images are becoming more widespread and effective self-presentation is becoming more challenging.

\subsection{Self-presentation challenges}

Traditionally, research contends that the affordances of $\mathrm{CMC}$ enable users to present idealized images online. For example, the hyperpersonal communication model suggests that the reduced number of cues in CMC and asynchronous communication enable users to selectively present themselves [34]. Individuals are likely to disclose charming and appealing characteristics and conceal unattractive ones. This behavior results in optimized images of the self [34].

The advantage of selective self-presentation lies in users' control over the content and distribution of their personal information [22]. On traditional CMC platforms like online chat rooms, all information is self-provided. However, today's Internet-based communication tools like social network sites (SNS) make other-provided information increasingly available to others. A typical example of otherprovided information on SNS is the tagging of personal photographs. Users can digitally 'tag' friends that appear in shared photos by linking images directly to their friends' public profile pages. As a consequence, this information (the digital photograph) becomes visible to all members of that friend's network. However, this other-provided information on SNS reduces the level of control users have over the information that is publicly available about themselves. Because there may be a lack of consensus about whether it is appropriate to publicize these pictures, the content and publicity of images shared by others may present information contradictory to one's idealized image [3, 25, 27]. Further, warranting theory argues that other-provided information is credible because it is less susceptible to manipulation [35]. Therefore, it often has a greater impact opposed to self-provided information. However, motivated users can engage in protective self-presentation to mitigate these problems.

\subsection{Protective self-presentation}

The evidence reviewed above suggests otherprovided information can have detrimental effects on the strategic self-presentation goals of SNS users. The literature highlights a range of strategies for protective self-presentation in response to unwanted otherprovided information $[1,18,27]$. These protective selfpresentation strategies are classified into two categories [27]. The first are repudiative strategies. These are used to deny certain characteristics associated with individuals. Individuals can also opt for an 'innocence' defense, look to justify themselves, or make compensatory self-presentations [1, 18, 27]. The second option includes subtractive strategies, referring to removing unwanted information. Individuals can disassociate themselves from the tagged photos (untagging), or delete unpleasant posts on their profile pages [27].

In sum, based on the two-component model of impression management and CSW research [18], we adopt the personality approach and propose a theoretical framework for the current study. Individuals that exhibit public sphere CSW stake their self-esteem on public evaluations. Thus, impression-based goals such as social acceptance, identity confirmation, and attention seeking function as primary motivators for strategic self-presentation. Therefore, these users should be more strategic to manage other-provided information, and this relationship is moderated by discrepancies between actual and ideal selves. Thus,

$\mathrm{H} 1$ : There is a positive association between public sphere CSW and engaging in protective selfpresentation in response to unwanted other-provided information online.

H1a: This relationship is moderated by actual-ideal self-discrepancies.

Further, literature shows there are different domains of public sphere CSW [5, 8]. For example, competency CSW refers to specific abilities like academic competence; competition $\mathrm{CSW}$ refers to outdoing others; and approval CSW refers to the perception of others' esteem; appearance refers to self-evaluations of one's physical appearance. However, which of these public sphere CSWs best predicts managing otherprovided information is unknown. Thus, we propose the following research question:

RQ1: What domain of public sphere CSW has the greatest impact on engaging in protective selfpresentation in response to unwanted other-provided information?

\section{The network approach}

Another theoretical framework that explains self- 
presentation focuses on the external environment. Goffman's dramaturgical approach compares selfpresentation to acting in which individuals cater their verbal and nonverbal communication to their audiences' expectations [11]. In face-to-face communication, audience refers to those that individuals interact with and can directly observe behaviors. However, audience scope is much greater online. On SNS, all the information related to individual users is visible to their network members, regardless of whether it is self- or other-provided. If they set their profiles public and allow non-friend members to view those pages, the audience is even further. In the current study, we operationalize audience as individuals' online network members.

The first characteristic of audience that likely influences self-presentation behaviors is clearly its size. One possible outcome associated with large audiences is more interactions between profile owners and their network [27]. This leads to increased chances of receiving other-provided information as well as information inconsistent with the ideal image users want others to hold of them. Thus,

$\mathrm{H} 2$ : Online network size is positively associated with engaging in protective self-presentation in response to unwanted other-provided information online.

Audience diversity likely affects self-presentation as well. Nowadays SNS users have increasingly diverse social networks comprised of close friends, family members, acquaintances and strangers. This makes effective self-presentation more challenging because social spheres have different expectations about individuals' ideal selves. For example, a photo of an underage individual drinking alcohol may be acceptable to friends, but it would likely be inappropriate for their parents. This phenomenon, known as the multiple audience problem, is associated with diverse audiences due to the complexity of balancing expectations of multiple social spheres [3, 18]. Failure to do so can cause ineffective selfpresentation, relational tension, and even social rejection $[3,32]$. Thus,

H3: Online network diversity is positively associated with engaging in protective self-presentation in response to unwanted other-provided information online.

Further, we present the following research question to address the lack of knowledge about how specific CSW relate to protective behavior online:

RQ2: Which model better predicts protective selfpresentation in response to unwanted other-provided information online-the personality model or the network model?

\section{Culture and self-presentation}

\subsection{Nationality as culture}

In addition to the factors presented above, research provides a great deal of evidence about the influence of culture on self-presentation behavior. One useful typology for understanding cultural differences is proposed by Hofstede [16]. Culture is operationalized as a function of nationality that consists of four dimensions: individualism/collectivism, power distance, uncertainty, and masculinity. The dichotomy of individualism and collectivism is the most widely used. Differences between individualistic and collectivistic cultures are originated from how members define their relationships with others [16]. Members of individualistic cultures regard themselves as independent, so competition is encouraged and personal achievement is valued. On the contrary, members of collectivistic cultures see themselves as interdependent with each other. As a result, they give priority to group achievement.

Literature suggests that culture affects protective self-presentation through self-monitoring [26], defined as the extent to which individuals are sensitive to what is appropriate and what is not [27]. High self-monitors are more concerned about how they are perceived and thus are more sensitive to cues indicating social appropriateness [27]. As individualistic cultural members value personal achievement, they are found to demonstrate high levels of self-monitoring in research $[14,15]$. Therefore, individualistic cultural members should be more strategic in managing otherprovided information. Thus,

H4: Individualistic cultural identity is associated with protective self-presentation in response to unwanted other-provided information online, opposed to collectivistic cultural identity.

\subsection{Gender as culture}

Gender refers to the expectations society has on human behavior based on biological differences [10]. For example, research found gender differences in language use and communication behaviors [20], supporting the gender-as-culture construct. If gender is understood as a cultural variable, it may also affect online self-presentation behaviors in predictable ways.

Research found more vulnerability to criticism in women because of their greater concern over relationships [12]. Thus, women should be more concerned about others' comments on their appearance, which motivates them to manage other-provided information more strategically. Thus, 
H5: Females are more likely to engage in protective self-presentation in response to unwanted otherprovided information online, opposed to males.

\section{Methods}

\subsection{Sample}

An online survey was conducted in a large northeastern university and a university in Singapore during spring 2011. Convenient samples were used in both universities by recruiting participants from introductory communication classes. This population is among the heaviest users of SNS.

In total, 249 responses and 161 responses were received from the American and Singaporean samples respectively. In order to keep the same sample size between two cultural groups, we randomly selected 161 responses from the American sample, yielding a final sample of 322 participants.

Among all participants, 117 (36.3\%) were male and $205(63.7 \%)$ were female. 32\% (103) were freshmen, $39.8 \%$ (128) were sophomore, $13.7 \%$ (44) were junior, and $14.6 \%$ (47) were senior-level students. Over half of the sample reported to be Asian (55.6\%, 179), followed by Caucasian $(37 \%, 119)$, African American $(3.1 \%, 10)$, Hispanic $(.9 \%, 3)$ and "others" $(.9 \%, 3)$.

\subsection{Measures}

Public sphere $C S W$ was measured with a 15 -item 7point Likert scale $(1=$ strongly disagree, $7=$ strongly agree) [5]. Sample items are "I don't care what other people think of me", and "my self-esteem is influenced by how attractive I think my face or facial features are" $(M=4.62, S D=.80$, Cronbach's alpha $=.81)$.

An exploratory factor analysis was conducted of the 15-item public sphere CSW measure, using principal components analysis with varimax rotation. Initially, four factors were identified that have an eigenvalue greater than 1. Two cross-loading items were dropped, making the remaining items factored into three dimensions: appearance CSW $(M=4.75, S D$ $=1.11$, Cronbach's alpha $=.77)$, approval CSW $(M=$ $4.41, S D=1.52$, Cronbach's alpha $=.87)$, and competition CSW $(M=5.15, S D=1.13$, Cronbach's alpha $=.89$ ).

Actual-ideal discrepancy was measured by using the self-acceptance scale [29]. This 4-item 7-point Likert scale asks respondents to indicate the degree to which they agree or disagree with the listed statements ( $1=$ strongly disagree, $7=$ strongly agree). Sample items are "in general I feel confident and positive about myself", and "I like most aspects of my personality".
The result was reverse coded $(M=2.95, S D=1.11$, Cronbach's alpha $=.70$ ).

Online network size was measured by asking participants "how many total friends do you have?" (M $=587.28, S D=402.59)$. Although it is a single-item measure, previous research has demonstrated that SNS users can recall the size of their online networks accurately [23, 30, 31].

Online network diversity was measured with a 16item scale that accesses a range of social categories including family, close friends, and co-workers [21]. Although this scale was originally used to measure network size, it was used in a recent study to measure network diversity [3]. Diversity was operationalized as an additive index of these items $(M=8.69, S D=2.89)$.

Unwanted other-provided information was operationalized as photo tagging and wall posts initiated by someone other than the users themselves that resulted in discomfort. Questions included "Have you ever been unhappy with a photo in which you were tagged?", "Have you ever been unhappy with the content of a wall post that involved you?", and "Have you ever been unhappy with the content of a wall post that someone posted on your wall?" If respondents answered "yes" to any of these questions, they were then prompted to report the protective strategy of selfpresentation from a list of options as shown below.

Protective strategy of self-presentation was operationalized as strategies in response to unwanted other-provided information that help individuals maintain positive public images. Items were developed from related research [27]. Strategies for managing unwanted photo tagging include, "I asked my friend(s) to remove the photo" and "I untagged my connection with the photo myself." 208 respondents reported protective reactions to unwanted photo tagging. Most $(\mathrm{N}=180)$ untagged the photos themselves, and 20 participants asked their friends to remove the photos.

Strategies for managing unwanted wall posts from others include, "I asked my friend(s) to remove it" and "I added another post to the wall so I could comment about it." A total of 37 participants used a subtractive strategy by asking their friends to remove posts about them that appeared on their friends' profile, and 26 adopted a repudiative strategy by adding another post in self-defense. Strategies for managing unwanted wall posts posted on the participant's online profile include "I removed it" and "I added another post to the wall so I could comment about it." 111 respondents adopted a subtractive strategy by removing the wall post that appeared on their own profile pages, and 14 used a repudiative strategy by adding another post in selfdefense. Because these two items are similar, they were combined to create a new variable measuring general protective behavior in response to unwanted 
other-provided wall posts. In total, $45 \%$ of respondents $(\mathrm{N}=145)$ reported protective reactions to these unwanted wall posts.

\section{Results}

In order to test the personality approach, we conducted logistic regression by using the response to unwanted photo tagging as the dependent variable first. This variable was dichotomized $(0=$ no reaction; $1=$ reacted with protective strategies). Controlling gender $(0=$ male, $1=$ female $)$ and cultural identity $(0=$ Singaporean, 1 = American), we entered the three types of CSW (appearance, approval, and competition) one at each time as one of the independent variables, along with actual-ideal discrepancy and the interaction between each CSW and actual-ideal discrepancy. When appearance CSW was tested, the model performed the best $\left(\chi^{2}=40.71, \mathrm{p}<.001\right.$, Cox $\&$ Snell $\mathrm{R}^{2}=.12$, see Table 1$)$. Individualistic cultural identity $(\mathrm{B}=.62, \mathrm{p}<.05)$ and appearance $\mathrm{CSW}(\mathrm{B}=.66, \mathrm{p}$ $<.001)$ have significant relationships with engaging in protective strategies to manage unwanted photo tagging. When approval CSW was tested, the model was also significant $\left(\chi^{2}=18.01, \mathrm{p}<.01\right.$, Cox \& Snell $\mathrm{R}^{2}=.05$, see Table 2$)$. Individualistic cultural identity $(\mathrm{B}=.70, \mathrm{p}<.01)$ and approval CSW $(\mathrm{B}=.26, \mathrm{p}<.01)$ have a significant relationship with engaging in protective strategies to manage unwanted photo tagging. Finally, when competition CSW was tested, the model was still significant $\left(\chi^{2}=24.87, \mathrm{p}<.001\right.$, Cox $\&$ Snell $\left.\mathrm{R}^{2}=.07\right)$. Gender $(\mathrm{B}=.68, \mathrm{p}<.05$, see Table 3$)$, individualistic cultural identity $(\mathrm{B}=.75, \mathrm{p}$ $<.01)$ and competition CSW $(\mathrm{B}=.44, \mathrm{p}<.001)$ have a significant relationship with engaging in protective strategies to manage unwanted photo tagging. Therefore, all three models produced consistent

Table 1. Appearance CSW and protective self-presentation in response to unwanted photo tagging.

\begin{tabular}{|c|c|c|c|c|}
\hline & \multicolumn{4}{|c|}{$\begin{array}{l}\text { protective self-presentation in response to } \\
\text { unwanted photo tagging }\end{array}$} \\
\hline & $\mathrm{B}$ & $\mathrm{SE}$ & Wald & $\operatorname{Exp},[\mathrm{CI}]$ \\
\hline Constant & $-3.94 * *$ & 1.51 & 6.86 & .02 \\
\hline Gender & .37 & .27 & 1.90 & $1.45,[.86,2.45]$ \\
\hline Culture & $.62 *$ & .27 & 5.41 & $1.86,[1.10,3.12]$ \\
\hline $\begin{array}{l}\text { Appearance } \\
\text { CSW }\end{array}$ & $.66 * * *$ & .13 & 28.14 & $1.94,[1.52,2.48]$ \\
\hline $\begin{array}{l}\text { Actual-ideal } \\
\text { Dscrp }\end{array}$ & .32 & .48 & .46 & $1.38,[.54,3.52]$ \\
\hline CSW *Dscrp & -.08 & .11 & .55 & $.92,[.74,1.15]$ \\
\hline $\begin{array}{c}\text { Note: }{ }^{*} p< \\
\text { Confidence } \\
\text { American }\end{array}$ & $\begin{array}{r}5, * * * p< \\
\text { terval; } M \\
\text {; No rea } \\
s\end{array}$ & $\begin{array}{l}01 ; 1 \\
l e=0\end{array}$ & $\begin{array}{l}\text { del } \chi^{2}= \\
\text { emale }= \\
\text { reacted } \\
=1 .\end{array}$ & $\begin{array}{l}71^{* * *} ; C I= \\
\text { Singapore }=0, \\
\text { ith protective }\end{array}$ \\
\hline
\end{tabular}

Table 2. Approval CSW and protective selfpresentation in response to unwanted photo tagging.

\begin{tabular}{|c|c|c|c|c|}
\hline & \multicolumn{4}{|c|}{ protective self-, photo tagging } \\
\hline & $\mathrm{B}$ & $\mathrm{SE}$ & Wald & $\operatorname{Exp},[\mathrm{CI}]$ \\
\hline Constant & -1.68 & .94 & 3.17 & .19 \\
\hline Gender & .47 & .26 & 3.22 & $1.59,[.96,2.65]$ \\
\hline Culture & $.70^{* *}$ & .26 & 7.21 & $2.01,[1.21,3.35]$ \\
\hline $\begin{array}{l}\text { Approval } \\
\text { CSW }\end{array}$ & $.26^{* *}$ & .09 & 8.99 & $1.30,[1.10,1.54]$ \\
\hline $\begin{array}{l}\text { Actual-ideal } \\
\text { Dscrp }\end{array}$ & .18 & .32 & .32 & $1.20,[.64,2.27]$ \\
\hline CSW *Dscrp & -.05 & .08 & .41 & $.95,[.82,1.11]$ \\
\hline
\end{tabular}

Table 3. Competition CSW and protective selfpresentation in response to unwanted photo tagging.

\begin{tabular}{|c|c|c|c|c|}
\hline & \multicolumn{4}{|c|}{ protective self-presentation, photo tagging } \\
\hline & $\mathrm{B}$ & $\mathrm{SE}$ & Wald & $\operatorname{Exp},[\mathrm{CI}]$ \\
\hline Constant & -2.37 & 1.57 & 2.30 & .09 \\
\hline Gender & $.68 *$ & .26 & 6.51 & $1.96,[1.17,3.30]$ \\
\hline Culture & $.75 * *$ & .26 & 8.27 & $2.12,[1.27,3.54]$ \\
\hline $\begin{array}{l}\text { Competition } \\
\text { CSW }\end{array}$ & $.44 * * *$ & .11 & 15.00 & $1.55,[1.24,1.94]$ \\
\hline $\begin{array}{l}\text { Actual-ideal } \\
\text { Dscrp }\end{array}$ & -.01 & .44 & .00 & $.99,[.42,2.32]$ \\
\hline CSW *Dscrp & .04 & .10 & .20 & $1.04,[.87,1.26]$ \\
\hline $\begin{array}{r}\text { Note: }{ }^{*} p<.05, \\
=\text { Confidence } \\
\text { American }\end{array}$ & ${ }^{p} p<.01$ & $* p$ & $91 ; M$ & $\begin{array}{l}\text { el } \chi^{2}=24.87^{* *} ; \mathrm{CI} \\
=1 ; \text { Singapore }=0, \\
\text { with protective }\end{array}$ \\
\hline
\end{tabular}

support to $\mathrm{H} 1$ and $\mathrm{H} 4$, but H1a was not supported, and H5 was partially supported.

We then replicated the same analysis by using the response to unwanted other-provided wall posts as the dependent variable. This variable was also dichotomized $(0=$ no reaction; $1=$ reacted with protective strategies). Only the model with appearance CSW was significant $\left(\chi^{2}=16.91, \mathrm{p}<.01\right.$, Cox \& Snell $\mathrm{R}^{2}=.05$, see Table 4). Appearance CSW $(\mathrm{B}=.34$, $\mathrm{p}$ $<.01)$ exhibited a significant relationship with engaging in protective strategies to manage unwanted other-provided wall posts. The moderation effect of actual-ideal discrepancy was also significant $(\mathrm{B}=-.21$, $\mathrm{p}<.05$ ). Therefore, H1 and H1a were both supported with appearance CSW. In addition, in both models, appearance CSW had the greatest impact on engaging in protective strategies to manage unwanted photo tagging. Further, in order to understand the moderation effect, we recentered actual-ideal discrepancy and replicated the analysis to compare the impact of appearance CSW on the dependent variable between high discrepancy and low discrepancy. We found that 
this relationship was only significant when actual-ideal discrepancy was low $(\mathrm{B}=.54, \mathrm{p}<.01)$.

Table 4. Appearance CSW and protective selfpresentation in response to unwanted wall posts.

\begin{tabular}{|c|c|c|c|c|}
\hline & \multicolumn{4}{|c|}{ protective self-presentation, wall posts } \\
\hline & B & $\mathrm{SE}$ & Wald & $\operatorname{Exp},[\mathrm{CI}]$ \\
\hline Constant & $-2.69 * * *$ & .76 & 12.40 & .07 \\
\hline Gender & -.27 & .25 & 1.21 & $.76,[.47,1.24]$ \\
\hline Culture & .32 & .24 & 1.81 & $1.38,[.86,2.21]$ \\
\hline $\begin{array}{l}\text { Appearance } \\
\text { CSW }\end{array}$ & $.54 * *$ & .16 & 11.77 & $1.72,[1.26,2.35]$ \\
\hline $\begin{array}{l}\text { Actual-ideal } \\
\text { Dscrp }\end{array}$ & .94 & .51 & 3.35 & $2.55,[.94,6.94]$ \\
\hline CSW *Dscrp & $-.21 *$ & .10 & 3.93 & $.81,[.66,1.00]$ \\
\hline $\begin{array}{r}\text { Note: }{ }^{*} p<. \\
C I=\text { Confider } \\
\text { Americar }\end{array}$ & $\begin{array}{r}*^{*}<.01, \\
\text { nterval; } \\
\text { No react } \\
\text { stra }\end{array}$ & $\begin{array}{l}p<. \\
e=0\end{array}$ & i:Mo & $\begin{array}{l}l \chi^{2}=16.91 * * * ; \\
1 ; \text { Singapore }=0, \\
\text { th protective }\end{array}$ \\
\hline
\end{tabular}

In order to test the network approach, logistic regression was conducted by using the response to unwanted photo tagging as the dependent variable, online network size and diversity as independent variables, gender and cultural identity as control variables. The model was significant $\left(\chi^{2}=21.01, \mathrm{p}\right.$ $<.001$, Cox \& Snell $\mathrm{R}^{2}=.06$, see Table 5). Gender (B $=.66, \mathrm{p}<.05)$, individualistic cultural identity $(\mathrm{B}=.51$, $\mathrm{p}<.05)$, and online network diversity $(\mathrm{B}=.12, \mathrm{p}<.01)$ demonstrated a significant association with engaging in protective strategies to manage unwanted photo tagging. Therefore, $\mathrm{H} 2$ was not supported, but $\mathrm{H} 3$ to H5 were all supported.

The same analysis was replicated by using the response to unwanted other-provided wall posts as the dependent variable. The model was significant $\left(\chi^{2}=\right.$ 29.21, $\mathrm{p}<.001$, Cox \& Snell $\mathrm{R}^{2}=.09$, see Table 6). Online network size $(\mathrm{B}=.00, \mathrm{p}<.01)$ and diversity $(\mathrm{B}$ $=.13, \mathrm{p}<.01)$ demonstrated a significant association with engaging in protective strategies to manage

Table 5. Network variables and protective selfpresentation in response to unwanted photo tagging.

\begin{tabular}{lcccc}
\hline & \multicolumn{4}{c}{ protective self-presentation in response to } \\
& unwanted photo tagging & \\
\cline { 2 - 5 } & $\mathrm{B}$ & $\mathrm{SE}$ & Wald & Exp, [CI $]$ \\
\hline Constant & $-1.43^{* *}$ & .49 & 8.56 & .24 \\
Gender & $.66^{*}$ & .26 & 6.33 & $1.94,[1.16,3.24]$ \\
Culture & $.51^{*}$ & .25 & 3.99 & $1.66,[1.01,2.72]$ \\
Size & .00 & .00 & 3.08 & $1.00,[1.00,1.00]$ \\
Diversity & $.12^{* *}$ & .05 & 7.21 & $1.13,[1.03,1.23]$ \\
\hline Note: ${ }^{*} p<.05, * * p<.01, * * * p<.001 ;$ Model $\chi^{2}=21.01 * * * ;$ \\
CI $=$ Confidence Interval; Male $=0$, Female $=1 ;$ Singapore $=$ \\
0, American $=1 ;$ No reaction $=0$, reacted with protective \\
strategies $=1$.
\end{tabular}

Table 6. Network variables and protective selfpresentation in response to unwanted wall posts.

\begin{tabular}{lcccc}
\hline & \multicolumn{4}{c}{ protective self-presentation in response to } \\
& unwanted other-provided wall posts \\
\cline { 2 - 5 } & $\mathrm{B}$ & $\mathrm{SE}$ & Wald & Exp, $[\mathrm{CI}]$ \\
\hline Constant & $-2.12^{* * *}$ & .49 & 18.77 & .12 \\
Gender & -.04 & .25 & .03 & $.96,[.59,1.58]$ \\
Culture & .30 & .24 & 1.52 & $1.35,[.84,2.18]$ \\
Size & $.00^{* *}$ & .00 & 9.73 & $1.00,[1.00,1.00]$ \\
Diversity & $.13^{* *}$ & .04 & 9.74 & $1.14,[1.05,1.24]$ \\
\hline \multicolumn{4}{c}{ Note: ${ }^{* *}<<.01, * * * p<.001 ;$ Model $\chi^{2}=29.21^{* * *} ;$} \\
CI=Confidence Interval; Male $=0$, Female $=1 ;$ Singapore $=0$, \\
American $=1 ;$ No reaction $=0$, reacted with protective \\
strategies $=1$.
\end{tabular}

unwanted other-provided wall posts. Thus, $\mathrm{H} 2$ and $\mathrm{H} 3$ were supported, but $\mathrm{H} 4$ and $\mathrm{H} 5$ were not.

Finally, in response to RQ2, the personality model worked better for engaging in protective strategies to manage unwanted photo tagging because it produced higher $\chi^{2}$ and Cox $\&$ Snell $\mathrm{R}^{2}$ than the network model. The only exception was the model with approval CSW, which yielded a slightly lower value of $\chi^{2}$ and Cox \& Snell $\mathrm{R}^{2}$ than the network model. However, the network model worked better for engaging in protective strategies to manage unwanted otherprovided wall posts.

\section{Discussion}

This study is a response to a big challenge of effective self-presentation in the contemporary CMC environment. As SNS enables users to interact with their contacts, the abundance of other-provided information reduces users' control over the information about themselves and may cause detrimental effects on the ideal images individuals expect to hold. Two goals are identified in this research: to compare the personality approach and the network approach to strategic management of unwanted other-provided information, and to compare the impact of different domains of public sphere CSW on the same behavior.

Our research started with examining the personality approach to protective self-presentation in response to unwanted other-provided information. We found consistent evidence that individuals exhibiting high levels of public sphere CSW were more motivated to strategically manage other-provided information. Specifically, appearance CSW demonstrated the largest association with engaging in protective selfpresentation behaviors to manage unwanted otherprovided information. Individuals staking their selfesteem on appearance are more concerned about whether they are considered as physically attractive. 
Thus, they are more motivated to manage otherprovided information related to their physical images. In addition, this relationship is also valid in the context of managing unwanted other-provided wall posts. One explanation can be that these wall posts may contain comments on users' physical appearance. Because individuals exhibiting high levels of appearance CSW are concerned about whether they are considered physically attractive, they may be alert to any opinions regarding their appearance and more motivated to manage them.

Overall, our finding that appearance CSW demonstrated the strongest relationship with protective self-presentation suggests that concerns about physical attractiveness are the main motives of managing otherprovided information on SNS. This may be related to the fact that we used college students as our sample and over half of them are female. Due to social and cultural pressures of physical attractiveness, female college students may be more concerned about how others think of their appearance [6]. Further, being physically attractive is desirable for young adults, particularly in a context of attention competition. Thus, they are more motivated to engage in protective selfpresentation behaviors.

The moderation effect between public sphere CSW and actual-ideal discrepancy on strategic management of other-provided information was generally not supported with only one exception. One explanation can lie in our operationalization of actual-ideal discrepancy, which was measured as individuals' own acceptance of themselves. However, this concept was conceptualized in the two-component model of impression management as the distance between the ideal and the actual images perceived by others. Regardless, the fact that the majority of online network members is weakly connected ties may render this discrepancy less important to individuals.

We found the relationship between appearance CSW and strategic management of other-provided wall posts was moderated by actual-ideal discrepancy. This relationship was only significant when individuals reported low levels of actual-ideal discrepancy. Therefore, protective self-presentation in response to other-provided wall posts only happens when individuals stake their self-esteem on physical appearance and are generally satisfied with it. Because these users are confident in their physical appearance, they believe they can fix their image through protective strategies when it is publicly denied, which then leads them to the behavior. However, if individuals lack confidence in their physical appearance, they may have internalized this fact. As a result, they may be less sensitive to or have less intense reactions to any denial of their physical appearance.
The second part of our investigation focuses on the influence of network characteristics on protective selfpresentation behaviors. We consistently found a positive relationship between online network diversity and these behaviors. This lends support to the multiple audience problem [18] and the problem of conflicting social spheres in previous literature [3]. Because different audience members have different expectations, individuals need to make sure that their selfpresentation is appropriate to the entire audience. However, due to lack of consensus regarding what is appropriate to share, other-provided information may be inappropriate to certain segments of the audience. Therefore, individuals should be more motivated to manage this information when they have a diverse audience.

We also found a positive relationship between online network size and protective self-presentation in response to unwanted photo tagging. The extremely low beta coefficient suggests a weak relationship. Regardless, diversity has a stronger impact on strategic management of other-provided information.

One important finding comes from the comparison between the personality approach and the network approach. We found the personality approach had a stronger association with strategic management of photo tagging whereas the network approach worked better for predicting management of other-provided wall posts. One reason can be the different natures of photo sharing and wall post exchanging online. Although photo sharing is a technique of relationship maintenance, it can also be used for self-promotion and attention seeking [30]. Therefore, internal factors such as self-esteem and self-evaluations may explain those image-related behaviors better. However, as an example of one-to-one communication, wall post exchanging is a technique of relationship maintenance. Thus, individuals may be more concerned about others' reactions to the information disclosed in wall posts. As a result, external environments such as audiences may have a greater impact on this behavior.

We found general support to our two cultural hypotheses. First, individualistic cultural members were more motivated to manage other-provided information. This finding lent support to previous research that individualistic cultural members value personal success and are more sensitive to cues indicating social appropriateness. As personal success is important, they are more alert to other-provided information that may threat their success. Second, women were found more motivated to manage unwanted photo tagging. This result confirms the cultural and social norms that emphasize physical attractiveness for women. As a result of this pressure, 
women are more motivated to manage their appearance displayed publicly.

Several limitations are identified. First, audience size was measured by using single item. Although single-item measure can be unreliable, when precise and singular objects are measured, single items can produce similar reliability and predictive validity to multiple-item measures $[2,33]$. Second, culture was operationalized as nationality and dichotomous. Critics about this operationalization focus on the inability to assess cultural differences within countries [13]. Although this operationalization has been justified by a wide range of research, a more accurate alternative could be measuring participants' identification with more specific cultural dimensions [23].

In spite of these limitations, our study provides practical implications for self-presentation online. For example, as network diversity may cause the multiple audience problem, users need to take caution when presenting themselves to a diverse audience. They can choose private communication channels or make neutral statements. The group function, which Facebook and Google + have launched, can also be useful to segregate the audience.

In terms of theoretical implications, we found the personality approach and the network approach worked in different contexts. This finding suggests which approach works better depends on the communication mode. Therefore, future research should take the mode of Internet-based communication tools into account. Another important finding is that appearance CSW predicts management of other-provided information most, which may be related to the identity of the majority of our sample. Thus, this finding suggests personal identity can be an important factor that affects CSW and self-presentation behaviors, which future research can investigate.

Our research contributes to the understanding of self-presentation online in the recent $\mathrm{CMC}$ environment, particularly how individuals manage other-provided information to maintain positive public impressions. Both internal factors and network properties were found to affect self-presentation behaviors, depending on different contexts. Social and cultural norms that guide human behaviors offline still influence behaviors online through internal factors and external environments.

\section{References}

[1] R. Arkin, "Self-Presentation Styles", in J.T.Tedeschi (ed.), Impression Management Theory and Social Psychology Research, Academic Press, New York, NY, 1981, pp. 311-333.
[2] L. Bergkvist, and J. R. Rossiter, "The Predictive Validity of Multiple-Item Versus Single-Item Measures of The Same Constructs", Journal of Marketing Research, American Marketing Association, Chicago, IL, 2007, pp. 175-184.

[3] J. Binder, A. Howes, and A. Sutcliffe, "The Problem of Conflicting Social Spheres: Effects of Network Structure on Experienced Tension in Social Network Sites", Proceedings of the 27th International Conference on Human Factors in Computing Systems, ACM, New York, NY, 2009, pp.965974.

[4]. J. Crocker, "Contingencies of Self-Worth: Implications for Self-Regulation and Psychological Vulnerability", Self and Identity, Psychology Press, Florence, KY, 2002, pp.143149 .

[5]. J. Crocker, R. Luhtanen, M. Cooper, and A. Bouvrette, "Contingencies of Self-Worth in College Students: Theory and Measurement", Journal of Personality and Social Psychology, American Psychological Association, Washington, DC, 2003, pp. 894-908.

[6]. J. Crocker, and L. Park, "Seeking Self-Esteem: Construction, Maintenance, and Protection of Self-Esteem", in M. Leary, and J.P. Tangney (eds.), Handbook of Self and Identity, Gilford Press, New York, NY, 2005, pp. 291-313.

[7]. J. Crocker, S. Sommers, and R. Luhtanen, "Hopes Dashed and Dreams Fulfilled: Contingencies of Self-Worth and Graduate School Admissions", Personality and Social Psychology Bulletin, SAGE, Thousand Oaks, CA, 2002, pp. $1275-1286$.

[8]. J. Crocker, and C. Wolfe, "Contingencies of Self-Worth", Psychological Review, American Psychological Association, Washington, DC, 2001, pp. 593-623.

[9]. D. DeAndrea, N. Ellison, R. LaRose, C. Steinfield, and A. Fiore, "Serious Social Media: on the Use of Social Media for Improving Students' Adjustment to College", Internet and Higher Education, Elsevier, Maryland Heights, MO, 2012, pp. $15-23$.

[10]. A.H. Eagly, Sex Differences in Social Behavior: A Social-Role Interpretation, Erlbaum, Hillside, NJ, 1987.

[11]. E. Goffman, The Presentation of Self in Everyday Life, Anchor, New York, NY, 1959.

[12]. S. Gore, R.H., Aseltine, and M.E. Colten, "Gender, social-relationship involvement, and depression", Journal of Research on Adolescents, Wiley-Blackwell, Malden, MA, 1993, pp. 101-125.

[13]. E. Green, and D. Póez, "Variation of Individualism And Collectivism Within And Between 20 Countries: A Typological Analysis", Journal of Cross-Cultural Psychology, SAGE, Thousand Oaks, CA, 2005, pp. 321-335. 
[14]. W.B. Gudykunst, T. Nishida, K. Leung, G. Gao, M.H. Bond, G. Wang, and R.A. Barraclough, "A Cross-Cultural Comparison of Self-Monitoring", Communication Research Reports, Routledge, New York, NY, 1989, pp. 7-12.

[15]. W.B. Gudykunst, S. Yang, and T. Nishida, "Cultural Differences in Self-Consciousness and Self-Monitoring", Communication Research, SAGE, Thousand Oaks, CA, 1987, pp. 7-34.

[16]. G. Hofstede, Culture Consequences: International Differences in Work-Related Values, SAGE, Beverly Hills, CA, 1980.

[17]. J.M. Jellison, and K.A. Gentry, "Self-Presentation Interpretation of the Seeking of Social Approval", Personality and Social Psychology Bulletin, SAGE, Thousand Oaks, CA, 1978, pp. 227-230.

[18]. M. Leary, Self-Presentation: Impression Management and Interpersonal Behavior, Brown \& Benchmark's, Dubuque, IA, 1995.

[19]. C. Madge, J. Meek, J. Wellens, and T. Hooley. "Facebook, Social Integration, and Informal Learning at University: 'It is More for Socializing and Talking to Friends About Work Than For Actually Doing Work'", Learning, Media, and Psychology, Routledge, New York, NY, 2012, pp. 141-155.

[20]. D.J. Maltz, and R.A. Borker, "A Cultural Approach to Male-Female Miscommunication", in J.J. Gumpertz (ed.), Language and Social Identity, Cambridge University Press, Cambridge, 1982, pp. 195-216.

[21]. C. McCarty, P.D. Killworth, H.R. Bernard, E.C. Johnsen, and G.A. Shelley, "Comparing Two Methods for Estimating Network Size", Human Organization, Society for Applied Anthropology, Oklahoma City, OK, 2001, pp.28-39.

[22]. A. Ramirez, and J. Walther, "Information seeking and interpersonal outcomes using the Internet", in T.D. Afifi, \& W.A. Afifi (eds.), Uncertainty, Information Management, and Disclosure Decisions, Routledge, New York, NY, 2009, pp. 67-84.

[23]. D. Rosen, M.A. Stefanone, and D. Lackaff, "Online And Offline Social Networks: Investigating CulturallySpecific Behavior And Satisfaction”, Proceedings of IEEE's Hawaii International Conference on Systems Science (HICSS), IEEE Press, Los Alamitos, CA, 2010.

[24]. M. Rosenburg, Society and the Adolescent Self-Image, Princeton University Press, Princeton, NJ, 1965.

[25]. J. Rui, and M.A. Stefanone, "Strategic Image Management Online: Self-Presentation, Self-Esteem, and Social Network Perspectives", Paper Presented at the 62rd
Annual Conference of International Communication Association, Phoenix, AZ, 2011.

[26]. J. F. Schumaker, and R.A. Barraclough, "Protective Self-Presentation in Malaysian and Australian Individuals", Journal of Cross-Cultural Psychology, SAGE, Thousand Oaks, CA, 1989, pp. 54-63.

[27]. A. Smock, "Self-Presentation on Facebook: Managing Content Created by the User and Others", Paper Presented at the 60nd Annual Conference of International Communication Association, Singapore, 2010.

[28]. M. Snyder, "Self-Monitoring of Expressive Behavior", Journal of Personality and Social Psychology, American Psychological Association, Washington, DC, 1974, pp. 526537.

[29]. K.W. Springer, and R.M. Hauser, "An Assessment of the Construct Validity of Ryff's Scales of Psychological Well-Being: Method, Mode, and Measurement Effects", Social Science Research, Elsevier, 2006, pp.1080-1102.

[30]. M.A. Stefanone, and D. Lackaff, "Reality Television as a Model for Online Behavior: Blogging, Photo, and Video Sharing", Journal of Computer-Mediated Communication, Wiley-Blackwell, Malden, MA, 2009, pp. 964-987.

[31]. M.A. Stefanone, D. Lackaff, and D. Rosen, "Contingencies of Self-Worth and Social Networking Site Behavior", Cyberpsychology, Behavior, and Social Networking, Mary Ann Liebert, Inc., New Rochelle, NY, 2011, pp. 41-51.

[32]. R.S. Tokunaga, "Friend Me or You'll Strain us: Understanding Negative Events That Occur Over Social Networking Sites", CyberPsychology, Behavior, and Social Networking, Mary Ann Liebert, Inc., New Rochelle, NY, 2011, pp. 425-432.

[33]. J.P. Wanous, and M.J. Hudy, "Single-Item Reliability: A Replication and Extension", Organizational Research Methods, SAGE, Thousand Oaks, CA, 2001, pp. 361-375.

[34]. J.B. Walther, "Computer-mediated Communication: Impersonal, Interpersonal and Hyperpersonal Interaction", Communication Research, SAGE, Thousand Oaks, 1996, pp. $3-43$.

[35]. J.B. Walther, and M. Parks, "Cues Filtered out, Cues Filtered in: Computer-Mediated Communication and Relationships", in M.L. Knapp, \& J.A. Daly (eds.), Handbook of Interpersonal Communication, SAGE, Thousand Oaks, CA, 2002, pp. 529-563.

[36]. B.A. Woike, and A.H. Baumgardner, "Global-Specific Incongruencies in Self-Worth and the Search for SelfKnowledge", Personality and Social Psychology Bulletin, SAGE, Thousand Oaks, CA, 1993, pp. 290-295. 